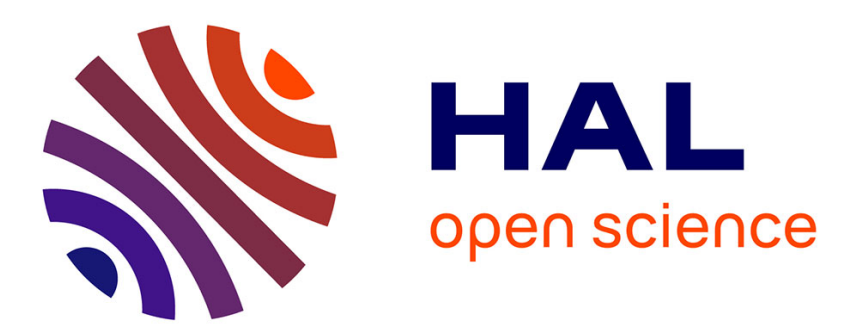

\title{
Identification of Nonlinear Volterra Models by Means of Diffusive Representation
}

\author{
Céline Casenave, Gérard Montseny
}

\section{To cite this version:}

Céline Casenave, Gérard Montseny. Identification of Nonlinear Volterra Models by Means of Diffusive Representation. 17th IFAC World Congress, Jul 2008, Seoul, South Korea. pp.4024-4029, 10.3182/20080706-5-KR-1001.00677 . hal-01061509

HAL Id: hal-01061509

https://hal.inria.fr/hal-01061509

Submitted on 7 Sep 2014

HAL is a multi-disciplinary open access archive for the deposit and dissemination of scientific research documents, whether they are published or not. The documents may come from teaching and research institutions in France or abroad, or from public or private research centers.
L'archive ouverte pluridisciplinaire HAL, est destinée au dépôt et à la diffusion de documents scientifiques de niveau recherche, publiés ou non, émanant des établissements d'enseignement et de recherche français ou étrangers, des laboratoires publics ou privés. 


\title{
Identification of Nonlinear Volterra Models by means of Diffusive Representation
}

\author{
C. Casenave* , G. Montseny* \\ ${ }^{*}$ LAAS-CNRS, University of Toulouse \\ 7 av. du Colonel Roche, 31077 Toulouse cedex 4, France. \\ e-mail: casenave@laas.fr, montseny@laas.fr
}

\begin{abstract}
We introduce a new identification method for nonlinear Volterra models of the form $\mathcal{H} x=f(u, x)$ with $\mathcal{H}$ a causal convolution operator. It is mainly based on a suitable parameterization of $\mathcal{H}$ deduced from the so-called diffusive representation, devoted to state representations of integral operators. Following this approach, the complex dynamic nature of $\mathcal{H}$ can be summarized by a few numerical parameters on which the identification of the dynamic part of the model will focus. For illustration, we implement this method on a concrete numerical example.
\end{abstract}

Keywords: Volterra model; non rational operator; state realization; diffusive representation.

\section{INTRODUCTION}

We introduce an identification method for nonlinear Volterra models of the form:

$$
H\left(\partial_{t}\right) x=f(u, x),
$$

where $u(t), x(t) \in \mathbb{R}, t>0, f$ is a sufficiently regular function defined on $\mathbb{R}^{2}$ and $H\left(\partial_{t}\right)$ is a causal convolution operator defined on a suitable space of continuous functions with support in $\mathbb{R}_{t}^{+}$.

We suppose that problem (1) is well-posed in the sense of existence, uniqueness and continuous dependency on the input $u$ of the solution $x$. The operator $H\left(\partial_{t}\right)$ is also supposed to be invertible, so (1) can be rewritten under the standard form: $x(t)=\int_{0}^{t} k(t-s) f(u(s), x(s)) d s \forall t>0$, where $k$ is the impulse response of operator $H\left(\partial_{t}\right)^{-1}$.

Such models are frequently encountered in various domains:

- thermic phenomena (we will consider in section 4 the combustion model elaborated in Joulin [1985]),

- electrical engineering (Rumeau [2006]),

- linear SISO differential systems with nonlinear feedback:

$$
\left\{\begin{array}{l}
\dot{X}=A X+B f(u, x) \\
x=C X
\end{array}\right.
$$

with $H(p)^{-1}=C(p I-A)^{-1} B$,

- SISO partial differential systems on $\mathbb{R}_{t}^{+*} \times \Omega_{z} \subset \mathbb{R}^{n+1}$ of the form:

$$
\left\{\begin{array}{l}
\partial_{t} \varphi=A(z, \nabla) \varphi+f\left(u, \int T \varphi d z\right), \varphi_{0}=0 \\
x=\int T \varphi d z
\end{array}\right.
$$

which can be rewritten under the synthetic equation $\int_{0}^{t} k(t-s) f(u(s), x(s)) d s=x(t)$ where the function $k$ is the impulse response associated with the operator $f \mapsto x$, that is the solution of:

$$
\left\{\begin{array}{l}
\dot{\phi}=A(z, \nabla) \phi+\delta, \phi_{0}=0 \\
k=\int T \phi d z
\end{array}\right.
$$

- etc.
The identification problem under consideration in the sequel is to build estimations (if possible optimal) of $H\left(\partial_{t}\right)$ and/or $f$ from (noised) data $x^{*}=x+v$ where $v$ designates some additive measurement noise and $x$ is generated by an experimental process driven by a known input $u$. The main difficulty when identifying such models results from the coupling between the dynamic operator $H\left(\partial_{t}\right)$ and the (static) function $f$ via equation (1). This difficulty is strengthened when operator $H\left(\partial_{t}\right)$ is non rational (which is in general the case), when $f$ is singular and when there exists some dynamic bifurcations (see section 4).

The proposed identification method is based on a suitable parameterization of $H\left(\partial_{t}\right)$ deduced from the so-called diffusive representation (Montseny [2005]), devoted to state realizations of integral causal operators. Following this approach, the complex dynamic nature of $H\left(\partial_{t}\right)$ is in some sense summarized by a few numerical parameters on which the identification problem will focus.

The paper is organized as follows. In section 2, we briefly present a simplified version of the diffusive representation. In section 3 , we describe the identification method in a general framework and we give some indications for numerical implementation. In section 4 , we finally implement this method on a concrete example, which allows us to comment the quantitative relevance of the method.

\section{DIFFUSIVE FORMULATION OF CAUSAL CONVOLUTION OPERATORS}

A complete statement of diffusive representation will be found in Montseny [2005]. Various applications and questions relating to this approach will be found for example in Audounet [1998], Carmona [1998], Casenave [2007], Casenave [2008], Degerli [1999], Garcia [1998], Lenczner [2005], Levadoux [2003], Montseny [2007], Mouyon [2002], Rumeau [2006]. 


\subsection{Mathematical framework}

We consider a causal convolution operator defined, on any continuous function $u: \mathbb{R}^{+} \rightarrow \mathbb{R}$, by

$$
u \mapsto \int_{0}^{t} k(t-s) u(s) d s .
$$

We denote $K$ the Laplace transform of $k$ and $K\left(\partial_{t}\right)$ the convolution operator defined by (2).

Let $u^{t}(s)=\mathbf{1}_{]-\infty, t]}(s) u(s)$ the restriction of $u$ to its past and $u_{t}(s)=u^{t}(t-s)$ the so-called "history" of $u$. From causality of $K\left(\partial_{t}\right)$, we deduce:

$$
\left[K\left(\partial_{t}\right)\left(u-u^{t}\right)\right](t)=0 \text { for all } t
$$

then, we have for any continuous function $u$ :

$$
\left[K\left(\partial_{t}\right) u\right](t)=\left[\mathcal{L}^{-1}(K \mathcal{L} u)\right](t)=\left[\mathcal{L}^{-1}\left(K \mathcal{L} u^{t}\right)\right](t) .
$$

We define:

$$
\Psi_{u}(t, p):=\mathrm{e}^{p t}\left(\mathcal{L} u^{t}\right)(p)=\left(\mathcal{L} u_{t}\right)(-p) ;
$$

by computing $\partial_{t} \mathcal{L} u_{t}$, Laplace inversion and use of (3), we have:

Lemma 1. 1. The function $\Psi_{u}$ is solution of the differential equation:

$$
\partial_{t} \Psi(t, p)=p \Psi(t, p)+u, \quad t>0, \quad \Psi(0, p)=0 .
$$

2 . For any $b \geqslant 0$,

$$
\left[K\left(\partial_{t}\right) u\right](t)=\frac{1}{2 i \pi} \int_{b-i \infty}^{b+i \infty} K(p) \Psi_{u}(t, p) d p .
$$

We denote $\Omega$ the holomorphic domain of $K$ (after analytic continuation). Let $\gamma$ a closed ${ }^{1}$ simple arc in $\mathbb{C}^{-}$; we denote $\Omega_{\gamma}^{+}$the exterior domain defined by $\gamma$, and $\Omega_{\gamma}^{-}$ the complementary of $\overline{\Omega_{\gamma}^{+}}$. By use of standard techniques (Cauchy theorem, Jordan lemma), it can be shown:

Lemma 2. For $\gamma \subset \Omega$ such that $K$ is holomorphic in $\Omega_{\gamma}^{+}$, if $K(p) \rightarrow 0$ when $p \rightarrow \infty$ in $\Omega_{\gamma}^{+}$, then:

$$
\left[K\left(\partial_{t}\right) u\right](t)=\frac{1}{2 i \pi} \int_{\tilde{\gamma}} K(p) \Psi_{u}(t, p) d p,
$$

where $\tilde{\gamma}$ is any closed simple arc in $\Omega_{\gamma}^{+}$such that $\gamma \subset \Omega_{\tilde{\gamma}}^{-}$.

We now suppose that $\gamma, \tilde{\gamma}$ are defined by functions of the Sobolev space $^{2} W_{\text {loc }}^{1, \infty}(\mathbb{R} ; \mathbb{C})$, also denoted $\gamma, \tilde{\gamma}$ and such that:

$$
\gamma(0)=0 .
$$

We use the convenient notation $\langle\mu, \psi\rangle=\int \mu \psi d \xi$; in particular, when $\mu$ is atomic that is $\mu=\sum_{k} a_{k} \delta_{\xi_{k}}$, we have: $\langle\mu, \psi\rangle=\sum_{k} a_{k} \psi\left(\xi_{k}\right)$.

Under hypothesis of lemma 2, we have (Montseny [2005]):

Theorem 3. If the possible singularities of $K$ on $\gamma$ are simple poles or branching points such that $|K \circ \gamma|$ is locally integrable in their neighborhood, then:

1. with $\tilde{\mu}=\frac{\tilde{\gamma}^{\prime}}{2 \mathrm{i} \pi} K \circ \tilde{\gamma}$ and $\tilde{\psi}(t,)=.\Psi_{u}(t,.) \circ \tilde{\gamma}$ :

$$
\left[K\left(\partial_{t}\right) u\right](t)=\langle\tilde{\mu}, \tilde{\psi}(t, .)\rangle ;
$$

\footnotetext{
1 Possibly at infinity

$2 W_{\text {loc }}^{1, \infty}(\mathbb{R} ; \mathbb{C})$ is the topological space of measurable functions $f$ : $\mathbb{R} \rightarrow \mathbb{C}$ such that $f, f^{\prime} \in L_{\text {loc }}^{\infty}$ (that is $f$ and $f^{\prime}$ are locally bounded in the almost everywhere sense).
}

2. with $^{3} \tilde{\gamma}_{n} \rightarrow \gamma$ in $W_{\text {loc }}^{1, \infty}$ and $\mu=\frac{\tilde{\gamma}^{\prime}}{2 \mathrm{i} \pi} \lim K \circ \tilde{\gamma}_{n}$ in the sense of measures:

$$
\left[K\left(\partial_{t}\right) u\right](t)=\langle\mu, \psi(t, .)\rangle,
$$

where $\psi(t, \xi)$ is solution of the following evolution problem on $(t, \xi) \in \mathbb{R}^{*+} \times \mathbb{R}$ (of diffusive type):

$$
\partial_{t} \psi(t, \xi)=\gamma(\xi) \psi(t, \xi)+u(t), \psi(0, \xi)=0 .
$$

Definition 4. The measure $\mu$ defined in theorem 3 is called $\gamma$-symbol of operator $K\left(\partial_{t}\right)$. The function $\psi$ solution of (10) is called the $\gamma$-representation of $u$.

Note in particular that thanks to (9), the Dirac measure $\delta$ is clearly a $\gamma$-symbol of the operator $u \mapsto \int_{0}^{t} u(s) d s$, denoted $\partial_{t}^{-1}$. We indeed have $\left(\partial_{t}^{-1} u\right)(t)=\langle\delta, \psi(t,)\rangle=$. $\psi(t, 0)$, with $\partial_{t} \psi(t, 0)=u, \psi(0,0)=0$.

Beyond the measure framework, the general space of $\gamma$ symbols is a quotient space of distributions, denoted $\Delta_{\gamma}^{\prime}$; it is the topological dual of the space $\Delta_{\gamma} \ni \psi(t,$.$) (Montseny$ [2005]). The composition product of operators has an equivalent in $\Delta_{\gamma}^{\prime}$, denoted $\sharp_{\gamma}$ or simply $\sharp$ : if $\mu$ and $\nu$ are respective $\gamma$-symbols of $H\left(\partial_{t}\right)$ and $K\left(\partial_{t}\right)$, then $\mu \sharp_{\gamma} \nu$ is a $\gamma$-symbol of $H\left(\partial_{t}\right) \circ K\left(\partial_{t}\right)$. Note that the product $\sharp_{\gamma}$ is inner, commutative and continuous ${ }^{4}$ in $\Delta_{\gamma}^{\prime}$ and so $\left(\Delta_{\gamma}^{\prime}, \sharp_{\gamma}\right)$ is an algebra (of $\gamma$-symbols) isomorphic to a commutative algebra of causal convolution operators.

Formulation $(10,9)$ can be extended to operators of the form $K\left(\partial_{t}\right) \circ \partial_{t}^{n}$ where $K\left(\partial_{t}\right)$ admits a $\gamma$-symbol in $\Delta_{\gamma}^{\prime}$. We have (formally):

$$
\left[K\left(\partial_{t}\right) \circ \partial_{t}^{n} u\right](t)=\left\langle\mu, \partial_{t}^{n} \psi(t, .)\right\rangle,
$$

with $\psi(t, \xi)$ solution of $(10)$ and $\mu$ the $\gamma$-symbol of $K\left(\partial_{t}\right)$. In the particular case where $n=1,(11)$ becomes:

$$
\left[K\left(\partial_{t}\right) \circ \partial_{t} u\right](t)=\langle\mu, \gamma \psi(t, .)+u(t)\rangle .
$$

In the same way, $\Delta_{\gamma}^{\prime}$ can be extended to an algebra denoted $\Sigma_{\gamma}$ whose elements are the $\gamma$-symbols of operators of the form $K\left(\partial_{t}\right) \circ \partial_{t}^{n}$ where $K\left(\partial_{t}\right)$ is associated to a $\gamma$-symbol $\mu \in \Delta_{\gamma}^{\prime}$ and $n \in \mathbb{N}$. $\gamma$-symbols $\nu$ of $K\left(\partial_{t}\right) \circ \partial_{t}^{n}$ are characterized by the relation:

$$
\mu=\nu \sharp \delta^{n}
$$

where $\delta^{n}=\underbrace{\delta \sharp \delta \sharp \ldots \sharp \delta}_{n \text { times }} \in \Delta_{\gamma}^{\prime}$ is a $\gamma$-symbol of $\partial_{t}^{-n}$.

The inversion of $\gamma$-symbols cannot be defined in $\Delta_{\gamma}^{\prime}$ because this algebra is not unitary; this operation is nevertheless well-defined in $\Sigma_{\gamma}$. If $\mu \in \Sigma_{\gamma}$ is a $\gamma$-symbol of $K\left(\partial_{t}\right)$ such that $K\left(\partial_{t}\right)^{-1} \circ \partial_{t}^{-n}$ has a $\gamma$-symbol $\nu \in \Delta_{\gamma}^{\prime}$, then $\nu=\mu^{-1} \sharp \delta^{n}$ and we have:

$$
\left[K\left(\partial_{t}\right)^{-1} u\right](t)=\left\langle\mu^{-1} \sharp \delta^{n}, \partial_{t}^{n} \psi(t, .)\right\rangle,
$$

with $\psi$ solution of (10). Note in particular that operator $\partial_{t}^{-1}$ defined above is the unique inverse of the derivative operator $\partial_{t}$.

\subsection{About numerical approximations}

We only give a few indications. More details can be found in the references cited above.

\footnotetext{
3 This convergence mode means that on any bounded set $P, \tilde{\gamma}_{n_{\mid P}}-$ $\gamma_{\mid P} \rightarrow 0$ and $\tilde{\gamma}_{n_{\mid P}}^{\prime}-\gamma_{\mid P}^{\prime} \rightarrow 0$ uniformly.

${ }^{4}$ In the sense of the strong topology of $\Delta_{\gamma}^{\prime}$.
} 
The state equation (10) is infinitedimensional. To get numerical approximations, we consider $\mathcal{M}_{L}$ a sequence of $L$ dimensional spaces of atomic measures on suitable meshes $\left\{\xi_{l}^{L}\right\}_{l=1: L}$ on the variable $\xi ; L$-dimensional approximations $\mu^{L}$ of the $\gamma$-symbol $\mu \in \Delta_{\gamma}^{\prime}$ are then defined in the sense of atomic measures, that is:

$$
\mu^{L}=\sum_{l=1}^{L} \mu_{l}^{L} \delta_{\xi_{l}^{L}}, \quad \mu_{l}^{L} \in \mathbb{C} .
$$

If $\cup_{L} \mathcal{M}_{L}$ is dense in the topological space $\Delta_{\gamma}^{\prime}$ (that is, concretely, if $\cup_{L}\left\{\xi_{l}^{L}\right\}$ is dense in $\mathbb{R}$ ), then we can have (Montseny [2005]):

$$
\left\langle\mu^{L}, \psi\right\rangle \underset{L \rightarrow+\infty}{\longrightarrow}\langle\mu, \psi\rangle \forall \psi \in \Delta_{\gamma}
$$

so, we have the following $L$-dimensional approximate state formulation of $K\left(\partial_{t}\right)$ (with $\gamma$-symbol $\mu$ ):

$$
\left\{\begin{array}{l}
\partial_{t} \psi\left(t, \xi_{l}^{L}\right)=\gamma\left(\xi_{l}^{L}\right) \psi\left(t, \xi_{l}^{L}\right)+u(t), l=1: L, \psi\left(0, \xi_{l}^{L}\right)=0 \\
{\left[K\left(\partial_{t}\right) u\right](t) \simeq \sum_{l=1}^{L} \mu_{l}^{L} \psi\left(t, \xi_{l}^{L}\right) .}
\end{array}\right.
$$

Note that an approximate state formulation of operator $\partial_{t} \circ K\left(\partial_{t}\right)$ can be easily deduced under the form:

$$
\partial_{t} \circ K\left(\partial_{t}\right) u \simeq \sum_{l} \gamma\left(\xi_{l}^{L}\right) \mu_{l}^{L} \psi\left(., \xi_{l}^{L}\right)+\sum_{l} \mu_{l}^{L} u .
$$

One of the properties of the approach presented above is that most of non rational operators encountered in practice can be closely approximate with small $L$ (see for example Montseny [2004]). In the context of identification of Volterra models, this will be a great advantage because only a few numerical parameters $\mu_{l}^{L}$ will have to be identified from experimental data, while the property (15) will ensure the well-posedness and the robustness of the problem as soon as the operator to be identified admits a $\gamma$-symbol in $\Sigma_{\gamma}$.

\section{IDENTIFICATION OF VOLTERRA MODELS}

In this section, we focus on the problem of identification of Volterra models of the form (1). We simply introduce the principle of the approach and give some indications about the numerical aspects of the problem. The statement is essentially formal. More details will be given in a further work.

\subsection{Principle}

In the sequel, we suppose for simplicity that the dynamic operators $H\left(\partial_{t}\right)^{-1}$ and $H\left(\partial_{t}\right) \circ \partial_{t}^{-1}$ both admit a $\gamma$-symbol in $\Delta_{\gamma}^{\prime}$; we denote $\mu$ any $\gamma$-symbol of $H\left(\partial_{t}\right) \circ \partial_{t}^{-1}$.

For any fixed $u$, equation (1) expresses the balance between two trajectories obtained from $x$, relating respectively to the linear dynamic operator $H\left(\partial_{t}\right)$ and the (nonlinear) static operator defined from function $f$ by ${ }^{5}:[f(u, x)](t)=$ $f(u(t), x(t)) \forall t$. So, from the point of view of trajectories (and if the numerical evaluation of $H\left(\partial_{t}\right) x$ is of reasonable cost), we can remark that the operators $H\left(\partial_{t}\right)$ and $f(u,$. play a comparable role in expression (1). The proposed

\footnotetext{
5 We distinguish carefully trajectories $(u, x, \ldots)$, which are functions of the time, and values taken by trajectories at time $t$ (i.e. $u(t)$, $x(t), \ldots)$
}

identification method then consists in parameterizing both operator $H\left(\partial_{t}\right)$ by means of its $\gamma$-symbol and function $f$ by means of a suitable functions basis. So, we will get an equivalent problem in which the unknown parameters are linearly dependent on the data and of reasonable dimension under numerical approximation (thanks to the properties of diffusive representation mentioned in the previous section).

Given a suitable $\gamma$ and denoting $\psi_{x}$ the $\gamma$-representation of $x$, according to (12), we consider the $\gamma$-realization of $H\left(\partial_{t}\right)$ defined by:

$$
H\left(\partial_{t}\right) x=\left\langle\mu, \gamma \psi_{x}\right\rangle+\langle\mu, 1\rangle x .
$$

By denoting $A_{x}$ the linear operator defined on any $\gamma$ symbol $\mu$ by:

$$
\forall t>0, \quad\left[A_{x} \mu\right](t)=\left\langle\mu, \gamma \psi_{x}(t, .)\right\rangle+\langle\mu, 1\rangle x(t),
$$

we then get: $H\left(\partial_{t}\right) x=A_{x} \mu$.

Now suppose that: $f=\tilde{f}+\bar{f}$ where $\tilde{f}$ is known a priori and $\bar{f}$ is unknown (to be identified from experimental data). We consider a topological basis $\left\{\mathbf{g}^{i} \otimes \mathbf{k}^{j}\right\}_{i, j=1:+\infty}$ of a tensorial product of Hilbert spaces to which belongs the function $\bar{f}$; we then have:

$$
f=\tilde{f}+\sum_{i, j} a_{i j} \mathbf{g}^{i} \otimes \mathbf{k}^{j},
$$

where the unknown is now the set of real parameters $a:=\left(a_{i j}\right)$. If the trajectories $u$ and $x$ are known (from measurements), so is it for the trajectories $\mathbf{g}^{i}(u), \mathbf{k}^{j}(x)$ and $\widetilde{f}(u, x)$; equation (1) can then be equivalently expressed under the linear form:

$$
A_{x} \mu-\sum_{i, j} \mathbf{g}^{i}(u) \mathbf{k}^{j}(x) a_{i j}=\tilde{f}(u, x) .
$$

By denoting

$$
\begin{gathered}
\mathcal{G}_{u, x}:(\mu, a) \mapsto A_{x} \mu-\sum_{i, j} \mathbf{g}^{i}(u) \mathbf{k}^{j}(x) a_{i j}, \\
b=\widetilde{f}(u, x), \quad \Xi=(\mu, a)
\end{gathered}
$$

and given two suitable Hilbert spaces $\mathcal{E}$ and $\mathcal{F}$, the identification problem of model (1) from data $\left(u, x^{*}\right)$, $x^{*}=x+v$ where $v$ designates some additive measurement noise, can be expressed as:

$$
\min _{\Xi \in \mathcal{E}}\left\|\mathcal{G}_{u, x^{*}} \Xi-b^{*}\right\|_{\mathcal{F}}^{2}
$$

Thanks to the linearity of operator $\mathcal{G}_{u, x^{*}}$, the solution of (19) is obtained by orthogonal projection:

$$
\Xi^{*}=\mathcal{G}_{u, x^{*}}^{\dagger} b^{*},
$$

where $\mathcal{G}_{u, x^{*}}^{\dagger}$ denotes the pseudo-inverse of $\mathcal{G}_{u, x^{*}}$ (Ben-Israel [2003]). In the sense of the hilbertian norm of $\mathcal{F}$, the estimation $\Xi^{*}$ of $\Xi$ is then optimal.

The physical system under consideration can then be described by the model $H^{*}\left(\partial_{t}\right) x=f^{*}(u, x)$ where $H^{*}$ and $f^{*}$ are deduced from the identified parameters $\mu^{*}, a^{*}$. Furthermore, we deduce from the equivalent expression $x=H^{*}\left(\partial_{t}\right)^{-1} f^{*}(u, x)$ that the following input-output state realization is available (up to the computation of $\delta \sharp\left(\mu^{*}\right)^{-1}$ which can be numerically performed) for simulation or control purposes:

$$
\left\{\begin{array}{l}
\partial_{t} \psi=\gamma \psi+f^{*}\left(u,\left\langle\delta \sharp\left(\mu^{*}\right)^{-1}, \psi\right\rangle\right), \psi(0, .)=0 \\
x=\left\langle\delta \sharp\left(\mu^{*}\right)^{-1}, \psi\right\rangle .
\end{array}\right.
$$




\subsection{Numerical formulation}

We consider now a finitedimensional subspace $\mathcal{E}_{k}$ of $\mathcal{E}$. After suitable time discretization and approximation of $\mu$ such as described in section 2 , we deduce from $(16,18)$ : $\forall n=1: N$,

$$
\begin{aligned}
{\left[\mathcal{G}_{u, x}\left(\mu^{L}, a\right)\right]\left(t_{n}\right)=} & \sum_{l=1}^{L} \mu_{l}^{L}\left(\gamma\left(\xi_{l}^{L}\right) \psi_{x}\left(t_{n}, \xi_{l}^{L}\right)+x\left(t_{n}\right)\right) \\
& -\sum_{i=1}^{I} \sum_{j=1}^{J} \mathbf{g}^{i}\left(u\left(t_{n}\right)\right) \mathbf{k}^{j}\left(x\left(t_{n}\right)\right) a_{i j}
\end{aligned}
$$

then the operator $\mathcal{G}_{u, x}$ can be expressed by means of a matrix $G_{u, x} \in \mathcal{M}^{N, L+I \times J}$ whose pseudo-inverse is classically given by ${ }^{6}$ (with $\left.N \gg L+I \times J\right)$ :

$$
G_{u, x}^{\dagger}=\left(G_{u, x}^{*} G_{u, x}+\epsilon I\right)^{-1} G_{u, x}^{*}
$$

Remark 5. Note that thanks to the state realization (10) of $\psi_{x}$ and the static nature of operators $\mathbf{g}^{i}, \mathbf{k}^{j}$, recursive formulations of (20) can be established, which allows to treat real-time identification or even pursuit problems.

\section{APPLICATION TO A MODEL OF FLAME}

In this section, we illustrate the identification method introduced above by implementing it on data $\left(u, x^{*}\right)$ elaborated from numerical simulations of a complex dynamic phenomenon studied in Joulin [1985], Audounet [1998].

\subsection{The model under consideration}

In Joulin [1985], Joulin elaborated a Volterra model to describe, in suitable thermodynamic conditions, the evolution of a spherical flame initiated by a source at point 0 in a mixture of reactive species. Under some reasonable physical hypothesis, such a phenomenon can be described by a system of two partial differential equations relating to the temperature and the mass density of the mixture. By considering the reactive zone as a thin sheet located on a sphere with radius $x(t)$, Joulin has established that when the flame is developing in free space, $x$ is solution of the following nonlinear singular Abel-Volterra equation ${ }^{7}$ $(u(t)$ designates the source strength at time $t)$ :

$$
x(t) \int_{0}^{t} \frac{\dot{x}(s) d s}{\sqrt{\pi(t-s)}}=2 x(t) \ln x(t)+2 u(t) \quad \forall t>0,
$$

with the additional conditions: $x(0)=0, u \geqslant 0, x \geqslant 0$ (whose physical interpretation is obvious). By denoting $H\left(\partial_{t}\right)$ the convolution operator ${ }^{8} x \mapsto \int_{0}^{t} \frac{\dot{x}(s) d s}{\sqrt{\pi(t-s)}}$ and $f(u, x):=2 \ln x+2 \frac{u}{x},(22)$ can be formally rewritten under the form (1). It has been shown in Audounet [1998] that the evolution problem (22) is well-posed, that is the solution $x$ exists, is unique and depends continuously on $u$.

In real conditions however, various perturbations are involved in the evolution of $x$ (due for example to the loss of spatial symmetries), and both the convolution operator

${ }_{6} G^{*}$ is the dual of $G$ with respect to the scalar product under consideration. As usual, $\epsilon \geqslant 0$ is devoted to numerical reconditioning.

7 Here adimensional for simplicity.

8 Note that in this ideal case, $H\left(\partial_{t}\right)=\partial_{t}^{\frac{1}{2}}$.
$H\left(\partial_{t}\right)$ and the function $f$ will be more or less far from the ideal ones. So, an identification process can be justified if accuracy of the model is required.

We will consider in the sequel the problem of identification of (1) from data $\left(u, x^{*}\right)$ obtained from highly accurate numerical simulations of (22) (not described here).

\subsection{On the dynamic behavior of the flame radius}

It has been shown in Audounet [1998] that there exists a threshold relating to the power of the source $u$, beyond which the flame is developing whereas a quenching occurs below. In that sense, this evolution phenomenon is essentially unstable with two qualitatively different behaviors. Because of the hereditary nature of the problem, it is difficult to know the value of this threshold which must be evaluated on the basis of numerical simulations. In figures 1 and 2, the flame is either quenching or developing, the source function being given, as in Audounet [1998], by:

$$
u(t)=E t^{0.3}(1-t) \mathbf{1}_{[0,1]}(t)
$$

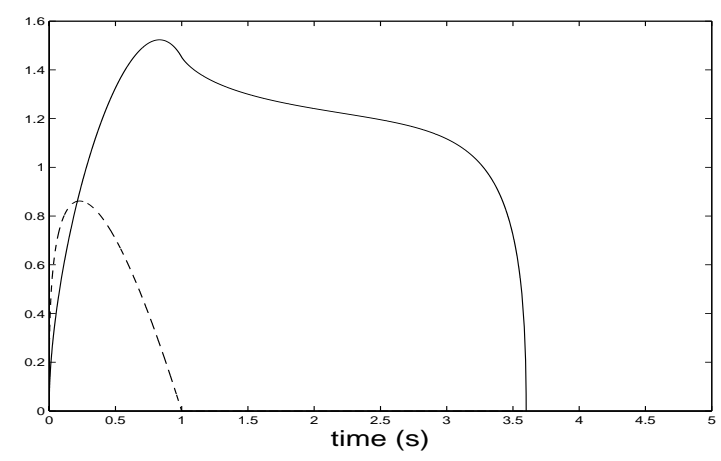

Fig. 1. Source (- - ) and radius of the flame $(-)$ with $E=1.7390$.

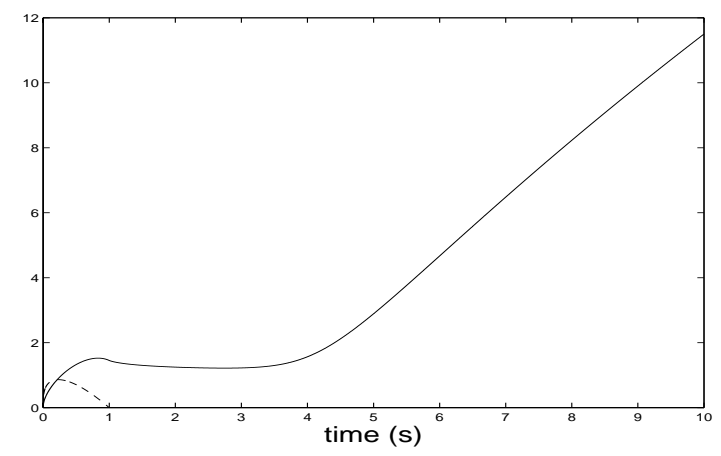

Fig. 2. Source (- - ) and radius of the flame (-) with $E=1.7393$.

Such dynamic behaviors generate ill-conditioned identification problems due to the sensitivity of the solutions $x$ of (1) with respect to the source power. As a consequence, this model can be viewed as a significant test for the proposed identification method.

\subsection{Parameterization of the problem}

The flame model under consideration can be written under the form (1) with, on the one hand: $f(u, x)=2 k(x)+2 \frac{u}{x}$ where the function $k$ is to be identified, and on the other 
hand, the operator $H\left(\partial_{t}\right)$ which is also to be identified with the assumption that $H\left(\partial_{t}\right) \circ \partial_{t}^{-1}$ admits a $\gamma$-symbol $\mu \in \Delta_{\gamma}^{\prime}$. As described in section 3 , we search an estimation of $f$ of the (simpler) form:

$$
f(u, x)=2 \frac{u}{x}+2 \sum_{j} a_{j} \mathbf{k}^{j}(x),
$$

where $\mathbf{k}^{j}$ are (known) basis functions by means of which the function $f(u, x)-2 \frac{u}{x}$ can be approximate, for example in the sense of $L^{2}\left(x_{\min }, x_{\max }\right)$. We simply consider power functions:

$$
\mathbf{k}^{j}(x)=x^{j-1} .
$$

According to section 3 , the vector $b^{*}$ is then defined by:

and the matrix $G_{u, x^{*}}$ by:

$$
b_{n}^{*}=2 \frac{u\left(t_{n}\right)}{x^{*}\left(t_{n}\right)}
$$

$$
\begin{gathered}
G_{u, x^{*}}=[\Psi \mid K], \\
\Psi_{n l}=\gamma\left(\xi_{l}^{L}\right) \psi_{x^{*}}\left(t_{n}, \xi_{l}^{L}\right)+x^{*}\left(t_{n}\right), \quad K_{n j}=2\left(x^{*}\left(t_{n}\right)\right)^{j-1} .
\end{gathered}
$$

\subsection{Numerical identification results and comments}

Recall that the problem consists in identifying the model (1) with the following functions to be estimated from numerical experimental data via the parameterizations $\mu, a$ defined above:

$$
H(p)=p^{\frac{1}{2}}, \quad f(u, x)=2 \ln (x)+2 \frac{u}{x} .
$$

In a first time, only the operator $H\left(\partial_{t}\right)$ is identified, while the function $f$ is suppose to be known. Then, $H\left(\partial_{t}\right)$ and $f$ are identified together.

The data are composed of 4 trajectories $\left(u, x^{*}\right)$ (see figure 3 ) associated with 4 different sources of the form ${ }^{9}$ (23) with $E=1.5,1.738,1.8$ and 5.0 respectively. The time step $\Delta t$ has been taken equal to $5 \cdot 10^{-5}$ and the maximal final time is equal to 10 .

The measured output is $x^{*}=x+\varepsilon v$ where $v$ is a unity gaussian white noise and $\varepsilon=10^{-3}$.

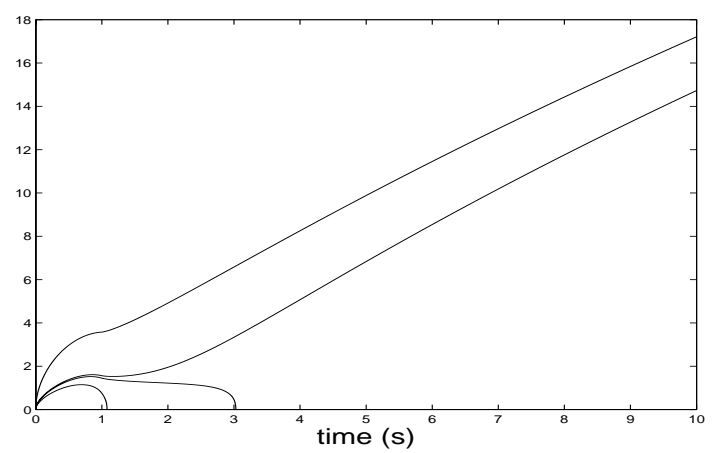

Fig. 3. The trajectories $x$ used for identification.

The function $\gamma$ is chosen as $\gamma(\xi)=\left\{\begin{array}{c}\xi e^{i \frac{3 \pi}{4}} \text { if } \xi>0 \\ -\xi e^{-i \frac{3 \pi}{4}} \text { if } \xi<0 .\end{array}\right.$

First, we suppose that $f$ is known and we identify $H\left(\partial_{t}\right)$ via the $\gamma$-symbol $\mu$. We use $L=20$ values $\xi_{l}^{L}$ geometrically

\footnotetext{
9 Note that the source function is physically realistic but rather poor from the point of view of information, which strengthen the difficulty of the problem.
}

spaced to cover 4 decades from $10^{-1}$ to $10^{3}$. The frequency response of the so-identified operator $H\left(\partial_{t}\right)$ is given in figure 4 . In the accessible frequency band, the identification is good. To complete the validation, we can compare in figures 5 and 6 the respective responses of the identified model and of the exact one for inputs $u$ different from those used for identification. In conclusion, when $f$ is known, the identified model closely behaves like the exact one.

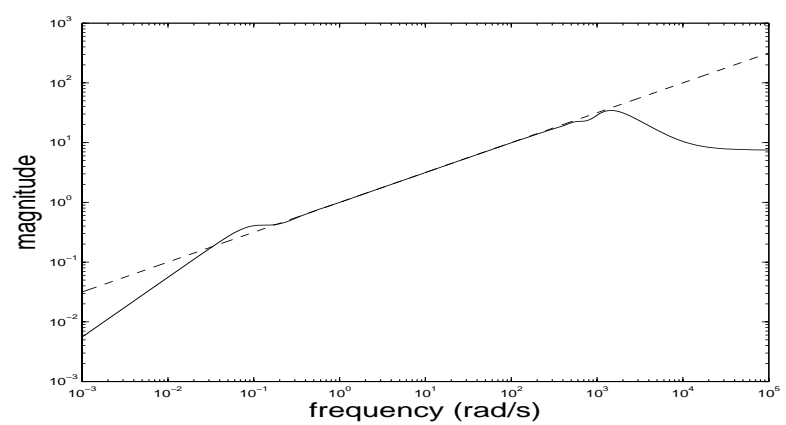

Fig. 4. Exact (- -) and identified (-) frequency responses $H(i \omega)$ when $f$ is known.

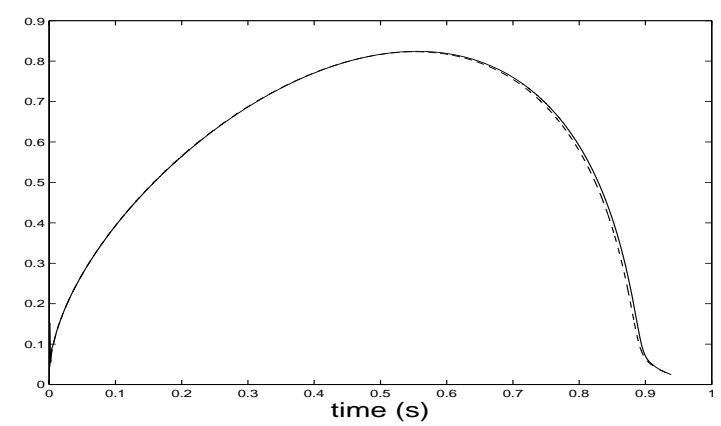

Fig. 5. Evolution of $x$ for the exact (- -) and identified (-) models with $u(t)=1.3 t^{0.3}(1-t) \mathbf{1}_{[0,1]}(t)$.

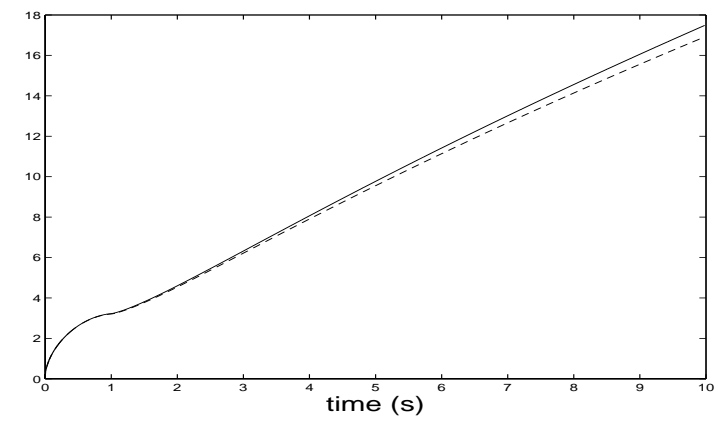

Fig. 6. Evolution of $x$ for the exact (- -) and identified (-) models, with $u(t)=4.0 t^{0.3}(1-t) \mathbf{1}_{[0,1]}(t)$.

We can add that results with comparable quality are obtained when $f$ is identified only (i.e. $H$ is supposed to be known).

We now consider the problem of identification of operator $H\left(\partial_{t}\right)$ and function $f$ together (both are supposed unknown). We use $L=20$ values $\xi_{l}^{L}$ geometrically spaced to cover 4 decades from $10^{0}$ to $10^{4}$ and the estimation of $f$ is searched as:

$$
f(u, x)=2 \frac{u}{x}+2 \sum_{j=0}^{9} a_{j} x^{j} .
$$




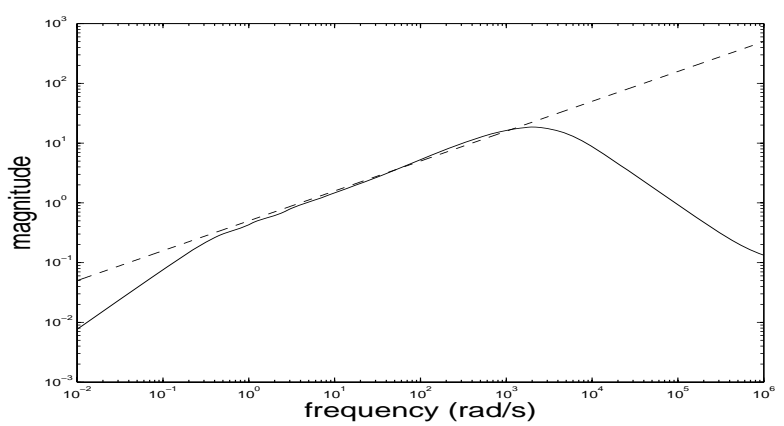

Fig. 7. Exact (- -) and identified (-) frequency responses $H(i \omega)$.

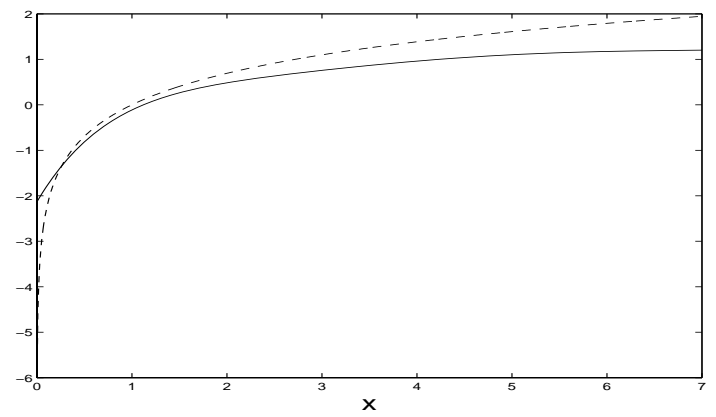

Fig. 8. Exact (- -) and identified (-) function $f$.

The so-identified operator $H\left(\partial_{t}\right)$ and function $f$ are given in figures 7 and 8 . The identified $H$ and $f$ remain close to the exact ones, which agrees with the theoretical analysis. However, due to the increasing number of parameters to be identified, the ill-conditioning of the problem and the dynamic poverty of the data $\left(u, x^{*}\right)$, the estimation error on $H$ and $f$ is greater than previously. It follows that the validation by simulation of the identified model reveals itself more delicate, in particular in the case of inputs with power close to the bifurcation threshold, which leads to the conclusion that more data will be necessary to improve the identification quality.

\section{CONCLUSION}

Relating to the first attempt to identify nonlinear Volterra models by use of the method presented in section 3 , the numerical results obtained in section 4 can be considered as positive, from both points of view of implementation simplicity and accuracy. Several questions must be studied in order to improve such results. For example, among the most significant, the involved hilbertian norms should be judiciously chosen and adapted to the specific properties of the class of models under consideration; indeed, this choice is crucial in terms of sensitivity with respect to perturbations of any nature. It can also be shown that the measurement noise induces some estimation bias which should be significantly reduced by appropriate treatments. All these questions are currently under study and will be discussed in a deepened paper.

\section{REFERENCES}

J. Audounet, J.M. Roquejoffre, An asymptotic fractional differential model of spherical flame, European Series on Applied and Industrial Mathematics (ESAIM): Proceedings, Vol.5, pp 15-28, Dec. 1998.

A. Ben-Israel, T.N.E. Greville, Generalized inverses: theory and applications, Springer-Verlag, New York, USA, 2003.

Y. Degerli, F. Lavernhe, P. Magnan, J. Farré, Bandlimited $1 / f^{\alpha}$ noise source, Electronics Letters, Vol. 35, no. 7, pp. 521-522, April 1999.

Ph. Carmona, L. Coutin, Fractional Brownian Motion and the Markov Property, Electronic Communications in Probability, Vol. 3, pp 95-105, Oct. 1998.

C. Casenave, E. Montseny, Time-local dissipative formulation and stable numerical schemes for a class of integrodifferential wave equations, To appear in SIAM Journal on Applied Mathematics.

C. Casenave, G. Montseny, Optimal identification of delay-diffusive operators and application to the acoustic impedance of absorbent materials, , IFAC Workshop on Time Delay Systems, Nantes, Sept. 17-19, 2007.

G. Garcia, J. Bernussou, Identification of the dynamics of a lead acid battery by a diffusive model, European Series on Applied and Industrial Mathematics (ESAIM): Proceedings, Vol.5, pp 87-98, Dec. 1998.

G. Joulin, Point-source initiation of lean spherical flames of light reactants: an asymptotic theory, Comb. Sci. and Tech., pp 99-113, 1985.

M. Lenczner, G. Montseny, Diffusive realization of operators solutions of certain operational partial differential equations, Comptes Rendus de l'Académie des Sciences (Mathématiques), Vol. 341 - No 12 - pp 737-740, Dec. 2005.

D. Levadoux, G. Montseny, Diffusive formulation of the impedance operator on circular boundary for $2 D$ wave equation, The Sixth International Conference on Mathematical and Numerical Aspects of Wave Propagation, June 30 - july 4, 2003, Jyväskylä, Finland.

G. Montseny, Diffusive representation for operators involving delays, in "Applications of time-delay systems", J.-J. Loiseau \& J. Chiasson eds. pp 217-232, SpringerVerlag, 2007.

G. Montseny, Représentation Diffusive, Hermes-Science, Paris, France, 2005.

G. Montseny, Simple approach to approximation and $d y$ namical realization of pseudodifferential time-operators such as fractional ones, IEEE Trans on Circ. \& Syst. II, Vol 51, No 11, pp 613-618, Nov. 2004.

G. Montseny, Diffusive representation of pseudodifferential time-operators, European Series on Applied and Industrial Mathematics (ESAIM): Proceedings, Vol.5, pp 159175, Dec. 1998.

Ph. Mouyon, N. Imbert, Identification of a $2 D$ turbulent wind spectrum, Aerospace Science and Technology, Vol 6, No 8, pp 599-605, Dec. 2002.

A. Rumeau, P. Bidan, T. Lebey, L. Marchin, B. Barbier, S. Guillemet, Behavior modeling of a $\mathrm{CaCu}_{3} \mathrm{Ti}_{4} \mathrm{O}_{12}$ ceramic for capacitor applications, IEEE Conference on Electrical Insulation and Dielectric Phenomena, Kansas City (Missouri USA), Oct. 15-18, 2006.

M. E. Taylor, Partial differential equations - II, Applied Mathematical Sciences, Springer, vol 116, 1997. 